\title{
ANÁLISIS COMPARATIVO DE LAS CRISIS DEL ECUADOR CON LAS DOS GRANDES CRISIS MUNDIALES DEL SIGLO XX Y LA CRISIS FINANCIERA DE INICIOS DEL SIGLO XXI
}

\section{COMPARATIVE ANALYSIS OF THE CRUISES OF ECUADOR WITH THE TWO BIG WORLD CRISIS OF THE XX CENTURY AND THE FINANCIAL CRISIS OF THE BEGINNINGS OF THE XXI CENTURY}

Karla Lissette Flores Cevallos, MSc. Máster en Economía y Desarrollo Territorial (España). Investigadora Directora Ejecutiva de la Fundación Los Andes, Ecuador. fundacionlosandes@yahoo.es

Carlos Ernesto Flores Tapia, Ph.D. Doctor en Ciencias Sociales y Jurídicas (España). Profesor Investigador de la Pontificia Universidad Católica del Ecuador, Ecuador. cflores@pucesa.edu.ec

\section{ARTÍCULO DE REVISIÓN BIBLIOGRÁFICA}

Recibido: 13 de diciembre de 2019.

Aceptado: 28 de enero de 2020.

\section{RESUMEN}

Las crisis económicas mundiales del siglo XX ocurrieron en la década de los años 30 y 70 y la crisis financiera de principios de este siglo, en el 2007. En el caso ecuatoriano, las crisis económicas mundiales tienen repercusiones negativas, no obstante, su historia se desarrolla a partir del año 1830, cuando se constituye como República Independiente. No obstante, los sucesivos gobiernos diseñan e implementan una serie de iniciativas en el ámbito económico con resultados que no logran aislar al Ecuador de los factores exógenos desfavorables para la sostenibilidad del desarrollo nacional derivados de las crisis 
mundiales. Este trabajo recurre a fuentes secundarias y bases de datos de organismos nacionales e internacionales para explicar las incidencias de las crisis mundiales en el Ecuador y a la aplicación del Test de Chow, para analizar el cambio estructural entre los años 1999 y 2014. El estudio concluye señalando que estas crisis han incidido en un marco de inestabilidad política porque los gobiernos de turno implementan políticas económicas sin la debida estrategia de Estado que permita mitigar o neutralizar los efectos negativos en el empleo, balanza comercial, tipo de cambio y PIB, principalmente.

Palabras clave: Crisis mundiales, Economía, Desarrollo, Cambio estructural, Econometría, Test de Chow.

\section{ABSTRACT}

The global economic crises of the twentieth century occurred in the decade of the 30s and 70s and the financial crises of the beginning of this century, in 2007. In the Ecuadorian case, the global economic crises have negative repercussions, however, its history develops from the year 1830, when it is constituted as an Independent Republic. However, successive governments design and implement a series of initiatives in the economic field with results that fail to isolate Ecuador from the unfavorable exogenous factors for the sustainability of national development derived from global crises. This work uses secondary sources and databases of national and international organizations to explain the incidents of the world crises in Ecuador and to the application of the Chow Test, to analyze the structural change between 1999 and 2014. The study concludes by pointing out that these crises have affected a framework of political instability because the governments of the day implement economic policies without the proper State strategy that allows mitigating or neutralizing the negative effects on employment, trade balance, exchange rate and GDP, mainly.

Keywords: World crises, Economy, Development, Structural change, Econometrics, Test de Chow. 


\section{INTRODUCCIÓN}

Las crisis económicas mundiales del siglo XX ocurren en la década de los años 30 y 70 y la crisis financiera de principios de este siglo, en el 2007. La Gran Depresión que se inicia en Estados Unidos en el año de 1929, quiebra el sistema multilateral de pagos y comercio (López, 2009; Naranjo, 2016). En la década de los 70, estalló la crisis del petróleo debido a que los países árabes reducen sus exportaciones de petróleo (Malavé, 1982; Martínez, Tornell, \& Westermann, 2004). Finalmente, la crisis de 2007 se debe al colapso de la burbuja inmobiliaria en Estados Unidos, provocando desconfianza en el sistema financiero internacional (Galanes, 2016; Gutiérrez, 2013; Stiglitz, Sen, Fitoussi, Sarkozy, \& Obiols, 2013).

En el caso ecuatoriano, las crisis económicas mundiales tienen repercusiones negativas, no obstante, su historia se desarrolla a partir del año 1830, cuando se constituye como República Independiente. El modelo económico vigente en la época fue el denominado primario-exportador, caracterizado por el auge cacaotero, permitió la inserción marginal del Ecuador en el mercado internacional a finales del siglo XIX (Acosta \& Guijarro, 2017; Acosta, 2006). Sin embargo, el Ecuador inicia su vida republicana con la carga de la deuda independentista, la misma que fue saldada recién en el año 1974 con los ingresos generados por la exportación de petróleo (Pareja, 2003).

Por su parte, el auge cacaotero llega a su fin en el año 1914, que coincide con el inicio de la Primera Guerra Mundial, debido a las fluctuaciones de las exportaciones de cacao, debido a los cambios en la demanda de este producto en los mercados internacionales, por ejemplo, de $77.3 \%$ en 1914 a $40.4 \%$ en 1918 , luego se incrementa a $71.3 \%$ en 1920 y vuelve a descender a $29 \%$ en 1930 . También por los problemas políticos internos en el país, por ejemplo, el levantamiento armado de las provincias de Esmeraldas y Manabí, fomentado por los seguidores del presidente Eloy Alfaro (1842-1912), un golpe de Estado denominado Revolución Juliana, entre otros. Esto obliga al gobierno a realizar emisión inorgánica de dinero, a incrementar su endeudamiento externo y a restringir las importaciones con el fin de incrementar el consumo local. No obstante, las medidas

ISSN: 1390-9320, Vol. 7, No. 1, febrero 2020 
adoptadas no fueron efectivas, viéndose truncadas nuevamente el desarrollo nacional (Acosta, 2006; Correa, 2009; Toscanini, 2011).

En este escenario, el país reproduce el efecto de las crisis más importantes de la historia con cierta peculiaridad; una manifestación de este caso se produce en la crisis del año 70, ya que el Ecuador fue uno de los países que se benefició del boom petrolero; sin embargo, años después la dependencia del factor petróleo y la mala administración de los gobiernos de la época desembocarían en recesión económica (González, Escobar, \& Vera, 2018). Ante este contexto histórico, cobra importancia, en este estudio, el análisis del impacto de las crisis de los años 30 y 70, así como la crisis del 2007 en el Ecuador. Consecuentemente, la presente investigación se estructura en cuatro apartados: el primero, es una breve revisión teórica sobre la implicación de las crisis globales en los países; el segundo, presenta la metodología que se utiliza en la investigación; en el tercero se exponen los resultados y discusión del estudio y, en el cuarto apartado, se establecen las conclusiones más relevantes del trabajo.

\section{REVISIÓN TEÓRICA}

El término "crisis económica" se define como un punto de inflexión en un ciclo o en las fluctuaciones de una economía de mercado, es el momento -en el tiempo- que un período de prosperidad da paso a uno de recesión (Samuelson, Nordhaus, Pavón, \& Blanco, 2010). Además, se considera que, una crisis no solamente surge por factores externos en el sistema, sino también se origina por la participación de elementos endógenos que convergen entre sí (Galanes, 2016). De acuerdo al filósofo Morin (1976) la crisis (...) está conformada por una constelación de nociones interrelacionadas: la idea de perturbación [...], la de progresión de las incertidumbres; la de parálisis y rigidez de aquello que constituía la flexibilidad organizativa del sistema, de los dispositivos de respuesta y regulación" (p.1).

Ahora bien, la separación conceptual entre las finanzas y la economía real en las últimas décadas provocaron que el enfoque teórico e histórico del tema se concentrara en el comportamiento de los mercados financieros (Rapoport \& Brenta, 2010). En efecto, tanto

ISSN: 1390-9320, Vol. 7, No. 1, febrero 2020 
Marx como Keynes mencionaban las posibles disociaciones entre sectores, por un lado, el sector productivo y, por otro lado, el sector financiero; situación capaz de desestabilizar el sistema económico en los países (Brue \& Grant, 2009).

Para Marx (1967), las crisis son soluciones momentáneas de las contradicciones existentes en un sistema, esto es, a partir del enfoque capitalista se tiende al desarrollo absoluto de las fuerzas productivas. Asimismo, tiene como objetivo conservar el valor del capital existente y su valoración como medida extrema. Por su parte, Keynes (2003) enfatiza en la importancia de los valores financieros como un intento de los productores para disminuir los riesgos asociados con la inversión productiva y, a su vez, destaca la fácil transferencia de estos. En esta línea, Niveau (1981, p.126) menciona que "El desarrollo del capitalismo industrial ha venido acompañado de una gran inestabilidad. A los períodos de prosperidad y de expansión de la producción han sucedido períodos de depresión y de paro".

Por otra parte, investigaciones más recientes sobre crisis económicas mundiales enfocan nuevos factores estructurales para dar lugar este proceso, en primer lugar, varios países con densamente poblados se encuentran en procesos de crecimiento acelerado (Stiglitz \& Rodríguez, 2007). En segundo lugar, se presenta la escasez de recursos naturales en los países (Edwards, 2013; López, 2010) y, finalmente, se detalla la exorbitante concentración de la riqueza en las economías desarrolladas (Naciones Unidas, 2018; Barcena \& Prado, 2014; Piketty, 2014). Por su parte, Acemoglu \& Robinson, 2012; Loayza, 2011; Stiglitz, 2012 evidencian que las políticas e instituciones y no la globalización son el principal determinante de la volatilidad excesiva y las crisis económicas.

\section{MATERIALES Y MÉTODOS}

Para la sistematización de los principales conceptos teóricos y para la construcción del análisis de este estudio se aplica la técnica bibliográfica (Hernández-Sampieri, Fernández, \& Baptista, 2014), basada en la búsqueda de información a partir de fuentes secundarias sustentadas en bases de datos como Web of Science, Latindex, Scielo, Dialnet, Redalyc, 
Google Académico, Scopus, entre otras, analizando y revisando las aportaciones de los autores en cuanto a las implicaciones de las crisis económicas más relevantes de la historia.

Además, se utiliza la técnica histórico dialéctica (Fattorelli \& Arias, 2008; Hidalgo, 1996) al momento de exponer y discutir los resultados resultantes del procesamiento de series temporales del Ecuador, basados en datos del Banco Central del Ecuador (BCE). Por su parte, para la identificación de cambio estructural en el país, se aplica el contraste de Chow (Gujaratí \& Porter, 2010; Wooldridge, 2010) en el periodo de 1990 al 2014. Se utiliza este tipo de contraste ya que se conoce un punto de ruptura, determinándose posiblemente un cambio estructural importante en la serie temporal.

\section{RESULTADOS Y DISCUSIÓN}

\subsection{Crisis de los años 30}

La crisis de los años 30 o la Gran Depresión ocurre por la caída de los precios en la bolsa de valores de Wall Street en el año 1929, provocando el cierre de empresas y bancos en Estados Unidos. "La deflación, la caída de la producción, la acumulación de stocks, el desempleo masivo, la contracción del comercio mundial y la ruptura del sistema de pagos internacionales marcaron la coyuntura en la mayoría de países capitalistas avanzados" (López, 2009, p. 2)

Asimismo, la crisis se extendió a Latinoamérica, por ejemplo, en Ecuador se observó una reducción de las exportaciones. En 1933 registró una disminución del 49\% del nivel de exportaciones de 1929, las importaciones también registraron una disminución de alrededor del 63\% de 1929 (Naranjo, 2016). Además, se presentó un deterioro de precios de exportación y términos de intercambio (Fernández, 1999). Los ingresos gubernamentales decrecieron, ya que dependían de los aranceles sobre las importaciones y de los impuestos generados por comercio externo. De la misma manera, el sistema monetario basado en el patrón oro se ve afectado, reduciendo la liquidez; lo que generó deflación hasta 1932 , después al dejar el patrón oro aumentó la liquidez lo que provocó altos índices 
inflacionarios. (Naranjo, 2016). En consecuencia, el gobierno ecuatoriano plantea la sustitución de importaciones para reducir los efectos de shock externos; sin embargo, el resultado es un incremento importante del endeudamiento externo (Castro, 1985; Maldonado, 2016). No obstante, la crisis en el Ecuador no afectó a toda la población, puesto que la mayoría de agentes económicos no estaban integrados a la economía de mercado y subsistían mediante trueques locales. "...la mayoría de ecuatorianos vivían en áreas rurales, tenían acceso a alimento, vivienda y pudieron evitar el impacto plano de la declinación económica" (Rodríguez, 1992, p. 200).

Posteriormente, concluida la Segunda Guerra Mundial, en la década de los 50, el Ecuador se convierte en el principal productor y exportador de banano, con ello el Estado orienta el gasto e inversión pública a la construcción de carreteras, ferrocarriles que lograron la integración nacional. Así también, se construyeron puertos; especialmente el Puerto de Guayaquil, centralizando la actividad exportadora. Sin embargo, en los años 60, Ecuador vivió un proceso de inestabilidad política tras la sucesión de varios presidentes constitucionales; la balanza comercial presentó déficit, debido al crecimiento de las importaciones y al declive de las exportaciones de banano (Acosta, 2006).

\subsection{Crisis de los años 70}

Por el contrario, en la década de los 70 la situación del país cambió, la extracción y exportación de petróleo revitalizó la economía; especialmente cuando los países árabes bloquean su exportación de petróleo a algunos países industrializados, provocando un reajuste en el precio del crudo en el mercado internacional, -el crudo de Oriente paso de 3,83 dólares por barril en 1973 a 13,14 dólares en 1914-. Sin embargo, el endeudamiento externo creció. ya que contaba con los ingresos del petróleo como garantía de pago (Acosta, 2006). En la década del petróleo -años 70-, el Banco Central del Ecuador controló el mercado oficial, con una paridad fijada en 25 sucres por dólar, además un mercado libre en el que el tipo de cambio fluctuaba en respuesta a la oferta y demanda (Oleas, 2013). 
Adicionalmente, en la década de los 80 , el escenario económico de Ecuador no era alentador, ya que se enfrentó a la crisis de la deuda externa en América Latina (Tabla 1). De acuerdo a Parodi Trece (2015), los principales factores que propagaron la crisis fue el sobreendeudamiento de los gobiernos de América Latina. El resultado fue una combinación de déficit fiscal y déficit en la balanza comercial, el exceso de préstamos de la banca comercial internacional, deterioro del entorno económico mundial; caída de los precios de materias primas y aumento de las tasas de interés internacionales, entre otros (Parodi, 2015). En esta línea, el choque petrolero y la política instaurada por Estados Unidos denominada "reaganomics" -recuperación de capitales por parte de Estados Unidos mediante el incremento sostenido de sus tasas de interés- provoca importantes flujos de capital en el Ecuador, en tal sentido el Estado adoptó un ajuste neoliberal (Radcliffe, 2012).

Tabla 1. Deuda Externa Total de Ecuador (1979-1991)

\begin{tabular}{|c|c|c|c|c|c|c|c|c|c|c|}
\hline & \multirow{3}{*}{$\begin{array}{l}\text { Saldo } \\
\text { inicial }\end{array}$} & \multicolumn{6}{|c|}{$\begin{array}{c}\text { Deuda externa total, 1979-1991 } \\
\text {-millones de dólares. }\end{array}$} & \multirow{3}{*}{$\begin{array}{c}\text { Ajustes } \\
\text { por tipo } \\
\text { de } \\
\text { cambio }\end{array}$} & \multirow{3}{*}{$\begin{array}{l}\text { Atrasos } \\
\text { de } \\
\text { intereses }\end{array}$} & \multirow{3}{*}{$\begin{array}{l}\text { Saldo } \\
\text { final }\end{array}$} \\
\hline & & \multicolumn{2}{|c|}{ Desembolsos } & \multicolumn{2}{|c|}{ Amortizaciones } & \multicolumn{2}{|c|}{ Intereses } & & & \\
\hline & & Efectivos & Totales & Efectivas & Totales & Efectivos & Totales & & & \\
\hline 1979 & 2.975 & 1.851 & 1.851 & 1277 & 1.277 & 322 & 322 & 6 & & 3.554 \\
\hline 1980 & 3.554 & 1.950 & 1.950 & 899 & 899 & 511 & 511 & -4 & & 4.601 \\
\hline 1981 & 4.652 & 2.768 & 2.768 & 1513 & 1.513 & 626 & 626 & -38 & & 5.868 \\
\hline 1982 & 5.870 & 2.025 & 2.691 & 1218 & 1.885 & 771 & 771 & -44 & & 6.632 \\
\hline 1983 & 6.633 & 940 & 2.563 & 204 & 1.770 & 715 & 752 & -45 & & 7.381 \\
\hline 1984 & 7.381 & 465 & 1.863 & 252 & 1.572 & 911 & 922 & -76 & & 7.596 \\
\hline 1985 & 7.596 & 742 & 1.931 & 323 & 1.495 & 840 & 840 & 79 & & 8.111 \\
\hline 1986 & 8.111 & 1.244 & 3.102 & 441 & 2.285 & 763 & 763 & 136 & & 9.063 \\
\hline 1987 & 9.063 & 1.134 & 1.784 & 639 & 1.286 & 322 & 322 & 298 & 477 & 10.336 \\
\hline 1988 & 9.858 & 983 & 1.924 & 1.100 & 1.892 & 337 & 472 & -140 & 919 & 10.669 \\
\hline 1989 & 9.750 & 1.013 & 1.686 & 712 & 1.316 & 463 & 533 & -44 & 1.456 & 11.533 \\
\hline 1990 & 10.077 & 606 & 833 & 736 & 890 & 557 & 631 & 278 & 1.924 & 12.222 \\
\hline 1991 & 10.298 & 775 & 782 & 791 & 798 & 499 & 499 & 86 & 2.435 & 12.802 \\
\hline
\end{tabular}

Fuente: Oleas (2013)

A partir del gobierno de Osvaldo Hurtado en 1981, se aplica una política recesiva, enfocada a reducir las variables consumo e inversión para garantizar la acumulación de capitales. Sin embargo, también aplica la "sucretización"1, mecanismo con el cual el Estado asume el

\footnotetext{
${ }^{1}$ Mecanismo con el cual el Banco Central del Ecuador asume la deuda externa que mantenía el sector privado nacional con la banca privada internacional, que alcanzaba a inicios de 1983 el $25 \%$
}

ISSN: 1390-9320, Vol. 7, No. 1, febrero 2020 
pago en dólares de la deuda privada y los empresarios pagan la deuda al Banco Central en sucres- generando pérdidas al Estado -la deuda privada tenía un valor aproximado de 650 mil millones de sucres convirtiéndose en mil millones de dólares-(Maldonado, 2016). Por otra parte, "En 1980, el 8.9\% del presupuesto general del Estado se destinaba al pago de la deuda externa. En 1987 la proporción subió al 27.3\%” (Acosta, 2006). El ajuste recesivo de la década provocó en 1988 un déficit presupuestario, sin embargo, la reducción de las importaciones $-22.9 \%$ medidas en dólares- a la par del incremento de las exportaciones de petróleo constituyó una balanza comercial positiva que alcanzó 619 millones de dólares (Maldonado, 2016).

En la coyuntura de la década de los 90, el Ecuador enfrentó una crisis bancaria, a causa de la liberalización financiera de 1994 y los efectos de la presencia del fenómeno de El Niño en 1997-1998 con pérdidas en la producción nacional estimadas en 2.869 millones de dólares (CEPAL, 1998). En 1998, el precio del petróleo descendió a 9,2 dólares por barril (40,3\% menos que en 1997) y el volumen exportado se redujo 6,1\% (Oleas, 2013), en el mismo año Ecuador experimentó un proceso de inestabilidad política, impidiendo solventar los casos de desajuste económicos (Banco Central del Ecuador, 1999). Además, las crisis financieras internacionales -Efecto tequila en México y Crisis Asiática-; generaron un deterioro en la balanza de pagos. En este sentido,

La crisis se manifestó en una vertiginosa expansión del desempleo, el subempleo y la pobreza. La tasa de desempleo abierto ascendió, en las tres principales ciudades del país del 8\% en 1998 al 17\% a mediados de 1999, mientras la pobreza urbana pasó del $36 \%$ al $65 \%$. La crisis produjo también una masiva migración internacional. Se estima que al menos 700.000 ecuatorianos han dejado el país a partir de 1998 (Cerdas, Jiménez, \& Valverde, 2006, p. 2).

del total de la Deuda Externa del país -alrededor de 1.628 millones de dólares-. Por su parte, los deudores privados debían pagar en sucres al Banco Central del Ecuador con una paridad cambiaria y una tasa de interés fijada a la fecha de celebración de sus contratos de deuda, operación que resultó perjudicial para el país (Fattorelli \& Arias, 2008).

ISSN: 1390-9320, Vol. 7, No. 1, febrero 2020 
Ante la problemática, las autoridades económicas adoptaron ciertas medidas: en 1999, feriado bancario -congelamiento de depósitos bancarios para evitar la fuga del capital-, creación de la Agencia de Garantías de Depósito y en el año 2000 se dolariza² oficialmente la moneda ecuatoriana -25.000 sucres equivalían a un dólar-. Estas medidas reactivaron la economía ecuatoriana, por ejemplo: el crecimiento del PIB -4,74\% en 1999 a 5,29\% en 2005 (BCE, 2019) (Figura 1) y la inflación con un promedio anual de 51,96 en 1999 a 2,17 en 2005 (BCE, 2019) (Figura 2). No obstante, la recuperación post dolarización fue el resultado del incremento en el precio del petróleo y las remesas de los emigrantes (Correa, 2009).

Figura 1. Producto Interno Bruto de Ecuador a precios constantes (1990 - 2017)

PIB (Millones de dólares de 2007)

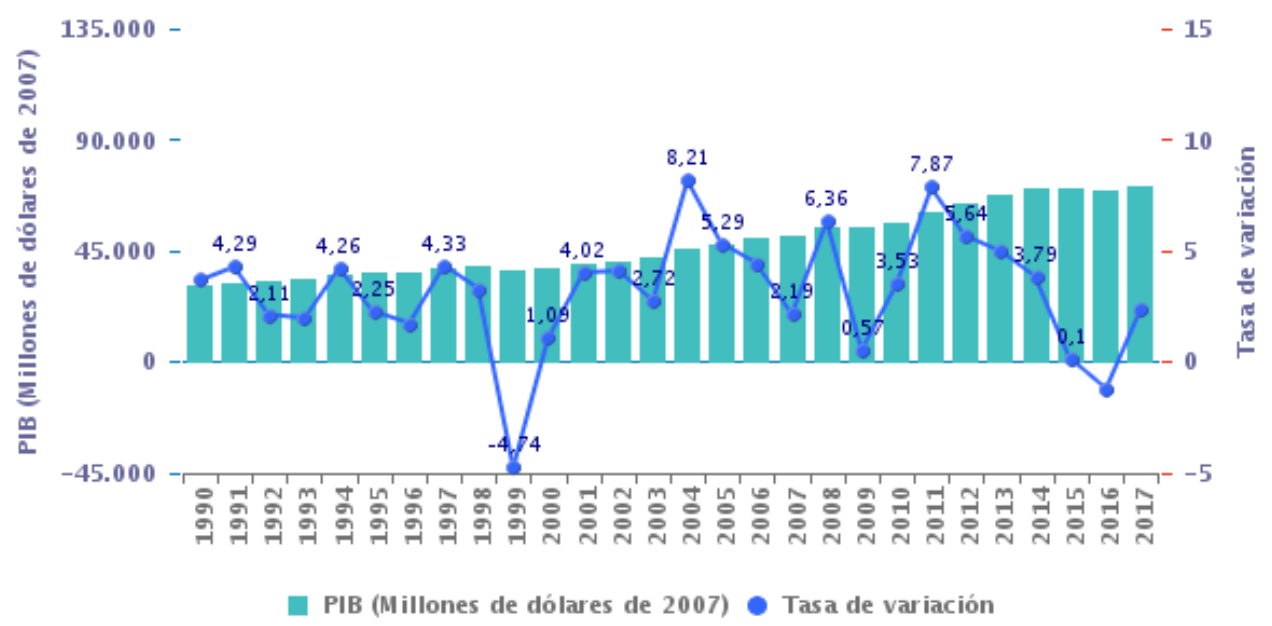

Fuente: Banco Central del Ecuador (2018)

\footnotetext{
${ }^{2}$ La dolarización es una medida mediante la cual el sucre es reemplazado por el dólar como reserva de valor, unidad de cuenta y como medio de pago y de cambio. La política monetaria pasa a depender de la Reserva Federal de los Estados Unidos de Norteamérica.
}

ISSN: 1390-9320, Vol. 7, No. 1, febrero 2020 
Figura 2. Variación porcentual de la inflación (1990 - 2015)

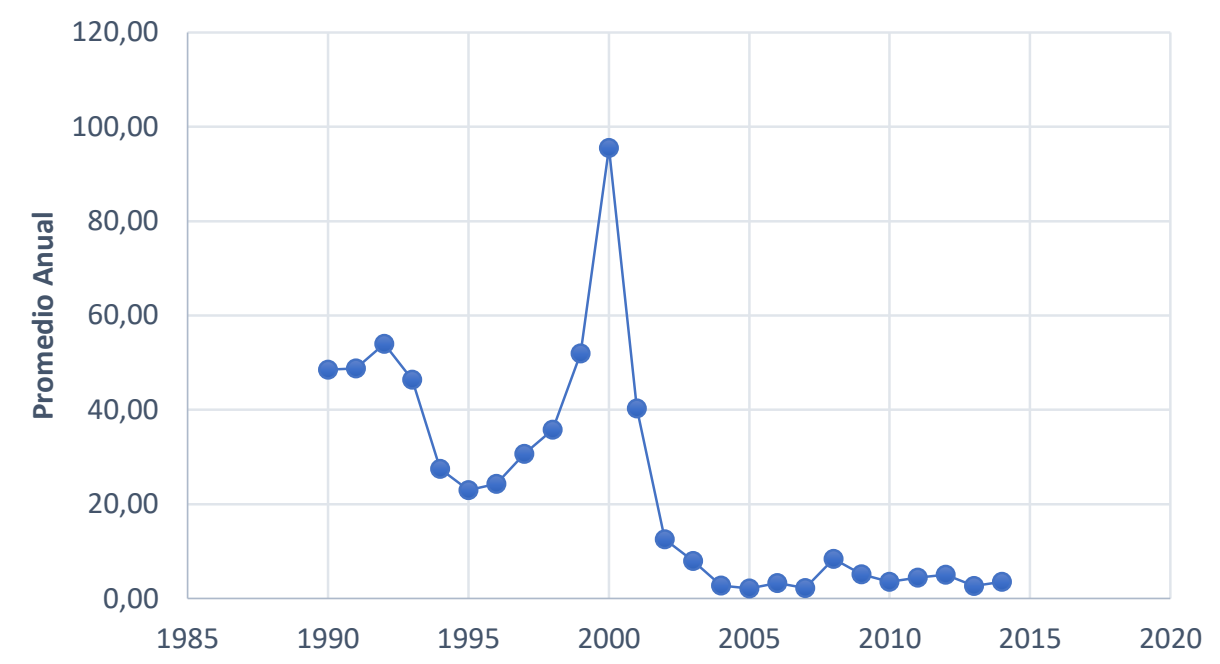

Fuente: Elaboración propia, a partir de datos del Instituto Nacional de Estadísticas y Censos (2018)

Al comparar las variables de serie de tiempo entre desempleo y Producto Interno Bruto PIB- en el Ecuador en los años 1990-2014, por medio del Test de Chow (Gujaratí \& Porter, 2010), los resultados arrojan en el periodo analizado un cambio estructural en los años 1999 y 2000 (Tabla 2).

Tabla 2. Test de Chow: Desempleo vs PIB en Ecuador

\begin{tabular}{|l|l|l|l|}
\hline Periodo de Tiempo & Valor P Variable Independiente & Cambio Estructural & Valor P \\
\hline $1990-2014$ & $0.0149 * *$ & Año 2000 & 0.0002 \\
\hline $1990-2000$ & $0.0521^{*}$ & Año 1999 & 0.0644 \\
\hline $1999-2014$ & $1.38 \mathrm{e}-05 * * *$ & Año 2000 & 0.5027 \\
\hline $2006-2014$ & $0.0102 * *$ & Año 2007 & 0.2565 \\
\hline
\end{tabular}

Fuente: Elaboración propia, a partir de datos Banco Central del Ecuador ( 2018) 


\subsection{Crisis financiera del 2007}

Según Ray \& Kozameh (2012), una nueva crisis económica mundial se presenta en el año 2007, cuando estalla la burbuja inmobiliaria de Estados Unidos, provocando en el Ecuador una recesión leve durante 2008-2009. Los efectos de la crisis fueron: caída del precio del crudo -44,6 dólares hasta mayo de 2009, en comparación a 99,7 dólares por barril en mayo de 2008-, las remesas cayeron en el 2008, en un 9,4\% respecto al 2007 -3.088 3 millones de dólares en el 2007 a 2.822 millones en el 2008-, en 2008 la inflación llegó a 8,4 \%, tres puntos porcentuales por debajo de la media de América Latina, entre otros (BCE, 2019). Sin embargo, la política económica utilizada por el gobierno ecuatoriano logró controlar la crisis, aplica una política fiscal expansiva, mantiene las tasas de interés bajas y restringe la cantidad de reservas de los bancos (Larrea, 2014).

Además, las políticas de gobierno implantadas desde 2007, condujeron a una nueva alternativa de desarrollo en el Ecuador, a partir de la política de transformación productiva mediante el cambio de la matriz productiva (Flores, Flores, Cuesta, \& Pérez, 2019; Flores \& Flores, 2015), sustentada en el Sumak Kawsay o Buen Vivir -que busca la igualdad de posibilidades y oportunidades, además de lograr un equilibrio con la naturaleza y la racionalidad económica reconciliándose con la ética y con el sentido común (Acosta \& Serrano, 2013). Nuevamente las acciones emprendidas se debilitan y son poco efectivas en el ámbito de la economía nacional, evidenciándose la persistencia de la debilidad institucional que incide en la sostenibilidad de la política pública y ratificando también la necesidad de que el enfoque de política pública no sea únicamente unidireccional de "arriba hacia abajo", sino también de "abajo hacia arriba", caso contrario, difícilmente serán sostenibles este tipo de políticas (Flores \& Flores, 2017; Appendini \& Nuijten, 2002; Pagés, 2010; Prokopenko, 1989).

\section{CONCLUSIONES}

Históricamente el Ecuador se ha visto afectado por varias crisis económicas mundiales, con consecuencias tales como el incremento del endeudamiento externo a finales de los años

ISSN: 1390-9320, Vol. 7, No. 1, febrero 2020 
20 y principios de los 30, en la década de los 80 y en los años 90 . A su vez, estas crisis han incidido en la inestabilidad política, porque los gobiernos de turno implementaron políticas económicas sin una estrategia de Estado que permita mitigar o neutralizar los efectos negativos en el empleo, balanza comercial, tipo de cambio y PIB, principalmente.

Antes de la década de los años 70, el modelo económico predominante era el agroexportador. Solo a partir del boom petrolero se dan los primeros pasos hacia el modelo de desarrollo industrial que permita mitigar las incidencias de los factores externos resultantes de crisis económicas como las explicadas en este estudio. Ahora bien, no es sino hasta el gobierno de Rafael Correa que se establece e impulsa estratégicamente una política de Estado enmarcada en los planes de desarrollo nacional (Secretaría Nacional de Planificación y Desarrollo, 2009), sistematizada en la Agenda de Transformación Productiva del Ecuador, teniendo como uno de los pilares de la diversificación de la producción, el cambio de la matriz productiva (Ministerio de Coordinación de la Producción Empleo y Competitividad, 2010). A su vez, el equipo que asumió el gobierno en mayo de 2017, bajo el liderazgo de Lenin Moreno, establece una estrategia económica y productiva que deja en manos del Frente Económico y del Consejo Consultivo Productivo Tributario (Presidencia de la República del Ecuador, 2017). Los primeros esfuerzos y resultados se recogen en el Informe sobre el Diálogo Nacional (Presidencia de la República del Ecuador, 2018); sin embargo, están aún pendientes sus resultados a favor de la sostenibilidad económica, social y ambiental (Flores, 2019; Pérez \& Castillo, 2017; Barroso, 2013).

ISSN: 1390-9320, Vol. 7, No. 1, febrero 2020 


\section{REFERENCIAS BIBLIOGRÁFICAS}

Acemoglu, D., \& Robinson, J. (2012). Por qué fracasan los países. Barcelona: Deusto. Acosta, A. (2006). Breve historia económica del Ecuador (1st ed.). Quito: Corporación Editora Nacional. Retrieved from http://www.flacsoandes.edu.ec/libros/digital/42390.pdf

Acosta, A., \& Guijarro, J. C. (2017). No somos idiotas : ; hace rato estamos en crisis ! Rebelión, 1-5. Retrieved from http://www.rebelion.org/noticias/2017/7/229159.pdf

Acosta, A., \& Serrano, A. (2013). Ecuador frente a un reto de internacional: la crisis económica múltiples aristas. Polemika, 8-24.

Appendini, K., \& Nuijten, M. (2002). El papel de las instituciones en contextos locales. Revista de La CEPAL, (Abril). Retrieved from http://www.cepal.org/es/publicaciones/10802-papel-instituciones-contextos-locales Banco Central del Ecuador. (1999). La economía ecuatoriana en 1999. Natural Language Engineering (Vol. 11).

Barcena, A., \& Prado, A. (2014). La hora de la igualdad: Brechas por cerrar, caminos por abrir. Santiago de Chile: CEPAL. Retrieved from http://www.cepal.org/es/publicaciones/36699-la-hora-la-igualdad-brechas-cerrarcaminos-abrir

Barroso, M. (2013). La Teoría de Desarrollo Local. In Teorías y estrategias de Desarrollo Local (Vol. 84, pp. 487-492). Sevilla: Universidad Internacional de Andalucía.

BCE. (2019). Base de datos del Banco Central del Ecuador. Retrieved September 13, 2017, from https://www.bce.fin.ec/

Brue, S., \& Grant, R. (2009). Historia del pensamiento económico. México: Cengage Learning.

Castro, F. (1985). La cancelación de la Deuda Externa y el Nuevo Orden Económico Internacional como única alternativa verdadera. La Habana: Editora Política. Retrieved from https://www.amazon.es/cancelación-económico-internacionalalternativa-verdadera/dp/B0026SFISS

CEPAL. (1998). El Fenómeno del Niño: su naturaleza y riesgos asociados a su 
presencia recurrente. México.

Cerdas, E., Jiménez, F., \& Valverde, M. (2006). Crisis de Ecuador en los años 1999 2000. Aula de Economía, 1-8.

Correa, R. (2009). De banana republic a la no república. Bogotá D.C.: Debate.

Retrieved from

http://app.pucesa.edu.ec:9040/OPAC/Home/BusquedaSimpleEjemplares?searchStrin

$\mathrm{g}=$ Historia economica Ecuador\&nombrebib=Biblioteca Juan Pablo

II\&idbib $=1$ \& registros $=25$ \&page $=1$

Edwards, B. (2013). Guía básica de la sostenibilidad. Barcelona: Editorial Gustavo Gilli.

Fattorelli, M., \& Arias, H. (2008). Informe de cierre de auditoría del tramo de sucretización de deuda privada INFORME No. 5/8 de la Subcomisión Deuda Comercial de la CAIC. Quito. Retrieved from

https://es.scribd.com/doc/111153060/Sucretizacion-ecuatoriana

Fernández, G. (1999). Compartir la carga: un esquema para reducir la Deuda Externa. Apuntes de Economía, 6, 1-17.

Flores, L., Flores, C., Cuesta, G., \& Pérez, A. (2019). Sectores estratégicos para el cambio de la Matriz Productiva: perspectiva de la ciudadanía y de los empresarios de Tungurahua. Boletín de Coyuntura, 20(1), 20-24. Retrieved from http://revistas.uta.edu.ec/erevista/index.php/bcoyu/article/view/687

Flores Tapia, C. (2019). Propuesta de mejora de la productividad y competitividad territorial en el contexto del cambio de matriz productiva del Ecuador aplicada a la provincia de Tungurahua. Universidad de Cádiz.

Flores, C., \& Flores, L. (2015). Desarrollo de herramientas de simulación de matrices productivas óptimas para el crecimiento económico del Ecuador. Tegucigalpa: CEAT-UNAH. Retrieved from http://www.ceat-unah.org/files/procceding_2015/tc/350TC.pdf

Flores, C., \& Flores, L. (2017). Impactos económicos inducidos por la demanda final en sectores estratégicos para el cambio de la estructura productiva del Ecuador.

Panorama Económico, 25(3), 443-458. Retrieved from

http://revistas.unicartagena.edu.co/index.php/panoramaeconomico/article/view/2085/ 1707

ISSN: 1390-9320, Vol. 7, No. 1, febrero 2020 
Galanes, I. (2016). La crisis económica mundial: un concepto complejo con múltiples denominaciones. Revista Filología E Linguística Portuguesa, 18(1), 5-41.

González, J., Escobar, M., \& Vera, G. (2018). La dependencia petrolera del ecuador y su incidencia en la oferta exportable. Revista Observatorio de Economía Latinoamericana, 1-17.

Gujaratí, D. N., \& Porter, D. C. (2010). Econometría. México: McGraw-Hill. Retrieved from

http://uca.summon.serialssolutions.com/2.0.0/link/0/eLvHCXMwdV1LCslwEB1KXSi48 FPxU8ELtLT5NO1aLYIbC92Xtkku0BN4Pw9IprVVRDchCSQkYcjwZnhvACjxA_rT6i4ASKCJzISUoWMa6pppWRcCcoka6X10hvPMppfeWZBX_8On6jEkheaoaoBWWYOUYCuQHjaMgnn7zDKpQI46IM_kLhNB6QhJFOZ2cYtwz7uvxwl-k

Gutiérrez, R. (2013). La primera crisis financiera global del siglo XXI: origen, contención e implicaciones productivas y laborales. Economía Teoría Y Práctica, (39), 145-193. http://doi.org/10.24275/etypuam/ne/392013/gutierrez

Hernández Sampieri, R., Fernández, C., \& Baptista, P. (2014). Metodología de la Investigación. México: McGraw Hill. Retrieved from http://observatorio.epacartagena.gov.co/wp-content/uploads/2017/08/metodologia-dela-investigacion-sexta-edicion.compressed.pdf

Hidalgo, H. (1996). Introducción a la investigación social (2ed. ed.). Quito: Ryndex

Ediciones. Retrieved from

http://biblioteca.udla.edu.ec/client/es_EC/default/search/detailnonmodal/ent: $\$ 002 \uparrow \$ 00$ 2fSD_ILS\$002f0\$002fSD_ILS:1/ada;jsessionid=AAEC041352A8FE05A76F46E528E F163F?qu=Hidalgo\%2C+Hugo+Hern\%C3\%A1n\&ic=true\&te=ILS\&ps=300

Keynes, J. (2003). Teoría general de la ocupación, el interés y el dinero (4th ed.). México: Fondo de Cultura Económica.

Larrea, A. M. (2014). Pobreza y desigualdad en el Ecuador: un balance de 7 años de Revolución Ciudadana. Patria: Análisis Político de La Defensa, 1(2), 22-33.

Retrieved from http://www.cecies.org/imagenes/edicion_574.pdf Loayza, N. (2011). Volatilidad y crisis: tres lecciones para países en desarrollo. In Pasado y presente: de la Gran Depresión del siglo XX a la Gran Recesión del siglo XXI (pp. 383, [3]). Bilbao: Fundación BBVA. 
López, E. (2009). CRACK DE 1929: Causas, desarrollo y consecuencias. Revista Internacional Del Mundo Económico Y Del Derecho Volumen I, 1-16.

López, R. (2010). Crisis económicas mundiales, escasez de recursos ambientales. Cepal.

Malavé, H. (1982). La crisis petrolera internacional y su incidencia. Comercio Exterior, 32(8), 864-875.

Maldonado, L. (2016). Ecuador Deuda Externa. Universidad Técnica de Machala.

Martínez, L., Tornell, A., \& Westermann, F. (2004). Globalización, crecimiento y crisis financieras: Lecciones de México y del mundo en desarrollo. El Trimestre Económico, 71(282), 251-351. Retrieved from http://search.proquest.com/docview/220867617?accountid=14495

Marx, K. (1967). Capital. New York: International Publishers.

Ministerio de Coordinación de la Producción Empleo y Competitividad. (2010). Agenda para la transformación productiva. Quito: Ministerio de Coordinación de la Producción, Empleo y Competitividad. Retrieved from http://www.produccion.gob.ec/wpcontent/uploads/downloads/2012/07/Agenda_Productiva[1].pdf

Morin, E. (1976). La notion du crise. Communications, 25.

Naciones Unidas. (2018). Agenda 2030 y los Objetivos de Desarrollo Sostenible: una oportunidad para América Latina y el Caribe. Publicación de las Naciones Unidas (Vol. Mayo). Santiago de Chile: CEPAL. http://doi.org/10.1017/CBO9781107415324.004

Naranjo, C. (2016). Historia Económica La Gran Depresión en Ecuador, 1927-1937. Universidad Autónoma de Barcelona.

Niveau, M. (1981). Historia de los hechos económicos contemporáneos. Barcelona: Ariel.

Oleas, J. (2013). Ecuador 1972-1999: Del Desarrollismo Petrolero al Ajuste Neoliberal. Universidad Andina Simón Bolivar.

Pagés, C. (2010). La era de la productividad: Cómo transformar las economías desde sus cimientos. Washington D.C.: Banco Interamericano de Desarrollo. Retrieved from http://www.iadb.org/research/dia/2010/files/DIA_2010_Spanish.pdf

ISSN: 1390-9320, Vol. 7, No. 1, febrero 2020 
Pareja, M. (2003). Canje, Conversión y Reducción de la Deuda Pública: El caso ecuatoriano. Apuntes de Economía, 28(economía), 1-28.

Parodi, C. (2015). La Crisis de la Deuda en América Latina de la década de los ochenta. Universidad del Pacífico. Lima.

Pérez, M., \& Castillo, J. (2017). Desde la colonización hasta una decolonialidad con concienciación colectiva endógena: el caso de América Latina. Panorama Económico, 25(4), 577-600. Retrieved from http://revistas.unicartagena.edu.co/index.php/panoramaeconomico/article/view/2090/ 1712

Piketty, T. (2014). Capital in the Twenty-First Century. Cambridge: Belknap Harvard. Retrieved from http://piketty.pse.ens.fr/files/capital21c/pdf/G2.2.pdf

Presidencia de la República del Ecuador. (2017). Decreto de creación del Consejo Consultivo Productivo y Tributario. Quito: Registro Oficial. Retrieved from http://colegiodeeconomistas.org.ec/noticias/wp-content/uploads/2017/07/23-06-17pol-decreto-50.pdf

Presidencia de la República del Ecuador. (2018). Informe sobre el Diálogo Nacional. Quito: Presidencia de la República del Ecuador. Retrieved from http://www.presidencia.gob.ec/wpcontent/uploads/2017/11/InformeDialogoNacionalparaWeb.pdf

Prokopenko, J. (1989). La gestión de la productividad. Organización Internacional del Trabajo. Ginebra: Organización Internacional del Trabajo. Retrieved from http://staging.ilo.org/public/libdoc/ilo/1987/87B09_433_span.pdf

Radcliffe, S. (2012). Development for a postneoliberal era? Sumak kawsay, living well and the limits to decolonisation in Ecuador. Geoforum, 43(2), 240-249.

http://doi.org/10.1016/j.geoforum.2011.09.003

Rapoport, M., \& Brenta, N. (2010). La crisis económica mundial: ¿el desenlace de cuarenta años de inestabilidad? Problemas Del Desarrollo, 41(163), 7-30. http://doi.org/10.22201/iiec.20078951e.2010.163.23499

Ray, R., \& Kozameh, S. (2012). La economía de Ecuador desde 2007 Contenido. Center for Economic and Policy Research, 1-27.

Rodríguez, L. (1992). Las Finanzas públicas en el Ecuador (1830-1940). (E. B. C. del 
Ecaudor, Ed.). Quito.

Samuelson, P., Nordhaus, W., Pavón, L., \& Blanco, C. (2010). Economía: con

aplicaciones a latinoamerica (Vol. 19ª). México [etc]: McGraw Hill. Retrieved from http://uca.summon.serialssolutions.com/2.0.0/link/0/eLvHCXMwY2AwNtlz0EUrE4yNj E2A5aCZgTI45g50NG4ykGmSYmpmkpoIPnbbLcA0MNA4xNs0ELocqhh87nYSdBgjH 3TzhV5qMbzTmJ2fkQjuWOpCFltCd6zlAL0DuUvDwswS2LIgZmA1AtXaoPaSHrwmN zQDzTciWkrAigq8TRXYbwMduAaa2zM2h5zPA-eDTiUBugGp-nE

Secretaría Nacional de Planificación y Desarrollo. (2009). Plan Nacional del buen Vivir 2009-2013. Quito: SENPLADES. Retrieved from http://www.planificacion.gob.ec/wpcontent/uploads/downloads/2013/07/Plan_Nacional_para_el_Buen_Vivir_version_res umida_en_espanol.pdf

Stiglitz, J. (2012). El precio de la desigualdad: el 1 por ciento de la población tiene lo que el 99 por ciento necesita. Madrid: Taurus. Retrieved from http://uca.summon.serialssolutions.com/2.0.0/link/0/eLvHCXMwbV1La8MwDBZ9XHZ r6crabpA_kBCcOEnPbcNglwZ6L37la1kghdD_PykOYay92NhgY4GsTxJ6ACQiisN_M gFJjTBaiVQaTdlw1yrPTcZt-

LYuw4STk8ujrKrk9CWrPhyq7epu696N0XDniwjbwWj8aS6qMyxDH2zZZ6zVRI7vpVG Q7iLIGKaCIloZfh8NSE5ARRa

Stiglitz, J., \& Rodríguez, C. (2007). El malestar en la globalización. Madrid, España: Punto de Lectura. Retrieved from http://uca.summon.serialssolutions.com/2.0.0/link/0/eLvHCXMwbV1LCsIwEB38bNwp Kn4hF2ixTZrqWi2CGwvdlySmKFYFu_NaHsGLOWmKiArZJCFhBkLevGHyAkB9dZ83QmhUtLTmmG0kXEj-

R1KqXyWMcUpZcqkeKNdEMc02QZxVQ5VILrbskpjXM3PF64u3qTxdD2lklg6ttiyerG Wozv2Lw0MnLHVoekjRJkDv3INLOPA5RR

Stiglitz, J., Sen, A., Fitoussi, J.-P., Sarkozy, N., \& Obiols, I. (2013). Medir nuestras vidas: las limitaciones del PIB como indicador de progreso. Barcelona: RBA. Retrieved from

http://uca.summon.serialssolutions.com/2.0.0/link/0/eLvHCXMwbV1NawlxEB38uPSm qNTawv6BXWI22U3OtkuhFxe8S5JNUFoqGOjv78zulqleEwj5YMjLm8nMA8h5xtKbO6 GQjpch5MK6hhQLpTU-

ISSN: 1390-9320, Vol. 7, No. 1, febrero 2020 
mHWjQsM0cxTgrbayrvPdl6z771CxrbttezfGiZQvMh8vpPH7dDAtsUy7z5Z9xtoPbqfT0I D4tNFiCGOOEEUG_54RL1NCE-5

Toscanini, M. (2011). La evolución de la economía ecuatoriana durante los últimos 30 años (1st ed.). Guayaquil: Universidad Católica Santiago de Guayaquil.

Wooldridge, J. (2010). Introducción a la econometría: un enfoque moderno. México:

Cengage Learning. Retrieved from

http://uca.summon.serialssolutions.com/2.0.0/link/0/eLvHCXMwY2AwNtlz0EUrE4yMk 82NzCyMzIEN6DTQ8JhJorGxkWkyqEtibgw--

9ktwDQw0DjE2zSQiQF2_x0oiBJBV17opRbDRy2ANSSwUwc6ZRvYGQclZBc9eA1ta GRoYmiOOPEMdFs1sEFkBuyPWZoDWxvg40kg5-7A-

ZagaiU5EalacRNkYAFtNRBiYEotFmFQ9AQtGk8 